\title{
Functional Anatomy of Auditory Verbal Imagery in Schizophrenic Patients With Auditory Hallucinations
}

\author{
Sukhwinder S. Shergill, M.R.C.Psych. \\ Ed Bullmore, M.R.C.Psych. \\ Andrew Simmons, Ph.D. \\ Robin Murray, D.Sc. \\ Philip McGuire, M.D.
}

Objective: This study investigated the functional neuroanatomy of inner speech and auditory verbal imagery in schizophrenic patients predisposed to auditory hallucinations.

Method: Eight patients with schizophrenia with a history of prominent auditory hallucinations and six comparison subjects underwent functional magnetic resonance imaging while generating inner speech or imagining external speech.

Results: Patients showed no differences while generating inner speech but experienced a relatively attenuated response in the posterior cerebellar cortex, hippocampi, and lenticular nuclei bilaterally and the right thalamus, middle and superior temporal cortex, and left nucleus accumbens during auditory verbal imagery.

Conclusions: Patients with schizophrenia who were prone to auditory hallucinations show attenuated activation when processing inner speech in areas implicated in verbal self-monitoring. ognitive models propose that auditory verbal hallucinations are derived from inner speech misidentified as external by means of defective self-monitoring (1). This suggests that the functional neuroanatomy of monitoring inner speech may be abnormal in patients who are prone to auditory verbal hallucinations. Both covert articulation and imagining another person's speech involve the generation of inner speech, but imagining speech (auditory verbal imagery) places greater demands on verbal self-monitoring (2).

We used functional magnetic resonance imaging (fMRI) to compare the neural correlates of inner speech and auditory verbal imagery in patients with schizophrenia who were predisposed to hallucinations and comparison subjects. We predicted that 1 ) both patients and volunteers would show activation in the left inferior frontal gyrus or insula when generating inner speech (2) and 2) patients, in relation to comparison subjects, would show attenuated activation in the temporal cortices and supplementary motor area during auditory verbal imagery, reflecting defective monitoring of inner speech $(2,3)$.

\section{Method}

Eight dextral male patients with DSM-IV schizophrenia and a mean age of 32 years $(S D=10$, range $=19-47)$, a mean IQ of 113
$(\mathrm{SD}=11$, range $=94-124)$, and a mean length of illness of 9.5 years $(\mathrm{SD}=7$, range $=3-20)$ were studied. All had experienced prominent and frequent auditory hallucinations during previous exacerbation of their illness but were in remission at the time of study. All except one (who was medication-free) had been taking a stable dose of antipsychotic medication (six treated with atypical antipsychotics; one with typical) for at least 3 months. Comparison subjects were matched for gender, handedness, age (mean $=34$ years, $\mathrm{SD}=4$, range $=27-38$ ), and IQ (mean $=114, \mathrm{SD}=8$, range $=100$ 120 ), had no medical or psychiatric disorders, had no family history of psychiatric disorders, and were not receiving medication. Subjects were screened for their ability to perform the tasks required. All provided written informed consent.

Four active conditions were each compared with a baseline condition. All tasks required that subjects listen to single words presented every 3 seconds in a neutral voice that they had heard during screening. Each comparison involved 10 alternating 30second blocks of each condition, each lasting 5 minutes. Task order was counterbalanced across subjects.

At baseline subjects listened to the words. For the inner-speech condition, upon hearing each word, subjects silently articulated a sentence in the form "I like..." or "I like being...," ending with the presented word. The first-person imagery condition had the same procedure as the inner-speech condition, except subjects imagined the sentence spoken in their own voice. For secondperson imagery, subjects imagined a sentence in the form "You like..." or "You like being...," ending with the presented word and spoken to them in the voice heard during screening. The thirdperson imagery condition had the same procedure as the second- 
FIGURE 1. Brain Regions Showing Attenuated Activation, Relative to That of Comparison Subjects, During Perception of Auditory Verbal Imagery in Schizophrenic Patients Prone to Auditory Hallucinations ${ }^{\mathrm{a}}$

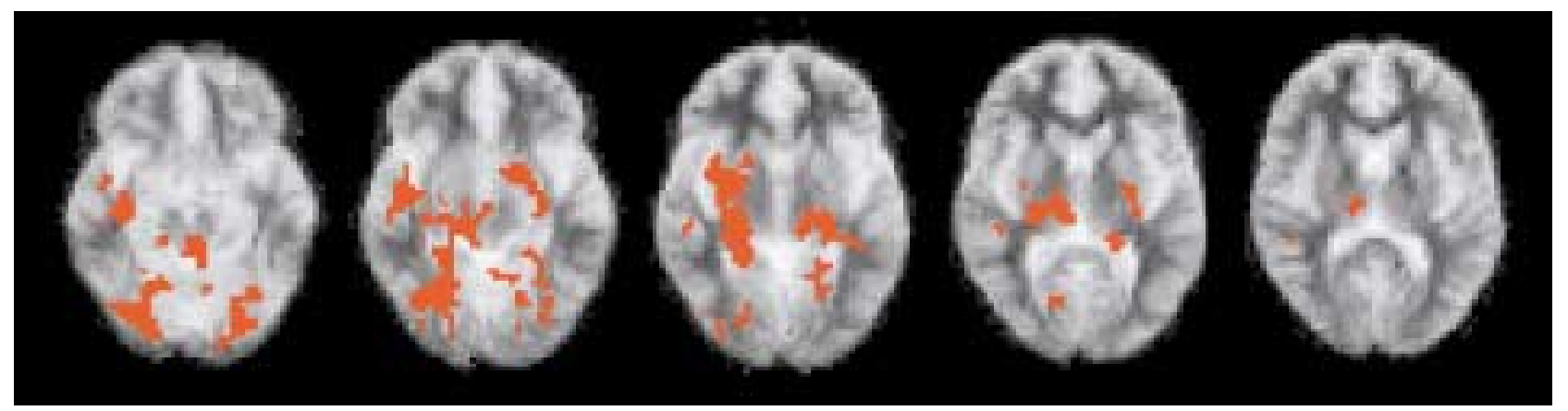

a Activation maps were rendered onto a template in Talairach space. Five axial slices, shown from left to right, represent the brain at $-17,-11$ $\mathrm{mm},-6 \mathrm{~mm}, 0 \mathrm{~mm}$, and $+6 \mathrm{~mm}$ relative to the intercommissural plane; the right side of each slice represents the left side of the brain. Attenuated activation (in red) is evident in the bilateral posterior cerebellar cortex (Talairach's coordinates $-10,-53,-11$, and $20,-47,-11$ ), hippocampi $(-24,-28,-6$, and $22,-14,-11)$, lenticular nuclei $(-28,-15,0$, and $25,-17,-6)$, right middle and superior temporal cortex (43, -10 , -11 , and $43,-31,0)$, thalamus $(15,-21,0)$, and left nucleus accumbens $(-22,-5,-11)$.

person condition, except that the sentence was in the form "He likes..." or "He likes being...." After completing each task subjects rated their ability to perform it as instructed by means of a visual analogue scale.

Gradient-echo echoplanar MR images were acquired by means of a 1.5-Tesla GE system (Milwaukee, modified by Advanced NMR, Wilmington, Mass.). In each of 14 noncontiguous planes in the intercommissural plane, $100 \mathrm{~T}_{2}$-weighted MR images depicting bold contrast were acquired (TE $=40 \mathrm{msec}$, $\mathrm{TR}=$ $3000 \mathrm{msec}$, in-plane resolution $=3.1 \mathrm{~mm}$, slice thickness $=7 \mathrm{~mm}$, slice skip $=0.7 \mathrm{~mm}$ ). The power of periodic signal change at the (fundamental) frequency of stimulation was estimated by the iterated least squares fitting of a sinusoidal regression model at each voxel. The fundamental power quotient (fundamental power divided by its standard error) was estimated at each voxel. If the median fundamental power quotient exceeded the critical value of the median fundamental power quotient obtained by randomization, for a one-tailed test of size $\alpha=2.5 \times 10^{-4}$, that voxel was considered generically activated with a probability of false positive activation of $\alpha$ (4).

Within-group activation maps were generated for the inner speech and the imagery tasks combined (representing auditory verbal imagery in general). Comparisons involving the individual imagery tasks addressed separate hypotheses and will be reported in the future. Between-group differences in activation during inner speech and auditory verbal imagery were estimated by fitting a one-way analysis of covariance model at each voxel to generate a map of the main effect of group at each voxel. This map was thresholded to generate a set of spatially contiguous three-dimensional clusters of suprathreshold voxel statistics, and the sum of suprathreshold voxel statistics in each cluster was tested against its sampled permutation distribution under the null hypothesis of zero group effect (see reference 5 for details).

\section{Results}

All patients reported they were able to perform the tasks during MRI scanning. There were originally eight comparison subjects, but two were excluded because they were unable to perform some of the imagery tasks during scanning (rating $=0 / 10$ ). There were no differences in the ratings of the patients and the remaining comparison volunteers. Only within-group activation for the patients (randomized $\mathrm{p}<2.5 \times 10^{-4}$ ) follows; results for the comparison subjects have been described elsewhere (3).

In patients, inner speech was associated with left-side activation in the inferior frontal gyrus or insula, precentral gyrus, superior temporal gyrus or inferior parietal lobule, and lingual gyrus. Activation was also evident in the supplementary motor area and the right posterior cerebellar cortex. An index of the consistency of activation across subjects, significant right cerebellar activation, was evident in five of the eight patients. During auditory verbal imagery (three imagery conditions combined) left-side activation was evident in the inferior and middle frontal gyri, pre- and postcentral gyri, and inferior parietal lobule. Further activation was evident in the supplementary motor area, right precentral and lingual gyri, and posterior cerebellar cortex.

Patients showed no differences in inner speech between groups (randomized $\mathrm{p}<0.001$ ). For auditory verbal imagery, patients showed attenuated activation in the posterior cerebellum, hippocampal complex, and lenticular nuclei bilaterally and also the right thalamus, middle and superior temporal gyri, and left nucleus accumbens (Figure 1).

\section{Discussion}

In this study we used fMRI to identify the neural correlates of tasks that engaged verbal self-monitoring in patients predisposed to auditory hallucinations. We minimized the potentially confounding effects of betweengroup differences in task performance by screening subjects. Although our patients were selected on the basis of their history of hallucinations, in the absence of a patient group not prone to hallucinations, we cannot exclude the possibility that the differential activation we observed was related to schizophrenia or medication, as opposed to the propensity to hallucinate. In addition, our modest group size limits how far one can generalize with these findings. 
As predicted, inner-speech generation was associated with activation in the left inferior frontal cortex or insula in both patients and comparison subjects. This is consistent with data from our previous positron emission tomography (PET) study and the notion that the generation of inner speech is not impaired in patients who are prone to hallucinations (2). Auditory verbal imagery is thought to entail retrieval of the voice to be imagined from memory and internal reproduction and inspection of speech in that voice (6). In the patients, auditory verbal imagery was associated with attenuated activation in the right temporal cortex. We predicted reduced left temporal activation because this was observed in our previous PET study (2). However, the attenuation of right temporal activation is consistent with other neuroimaging data implicating this region in verbal self-monitoring $(3,7)$ and in the experiencing of auditory hallucinations (8).

Patients showed an attenuation of activation in the cerebellum bilaterally, greater on the right, during the perception of auditory verbal imagery. Our previous study also found that hallucination-prone patients showed reduced cerebellar activation, relative to nonhallucinators, during the perception of auditory verbal imagery (2). Lesion and neuroimaging studies have suggested that the cerebellum normally acts as a comparator in both motor (9) and verbal tasks (10), comparing intended performance with actual performance and modulating cerebral cortical activity appropriately (11). During auditory verbal imagery, patients showed additional attenuated activations in the bilateral hippocampal complex, right thalamus, and left nucleus accumbens. These structures, in conjunction with the hippocampus, have also been proposed as the comparator in models of cognitive self-monitoring (12) and are activated during auditory hallucinations in schizophrenia $(8,13)$. However, they have also been associated with memory retrieval (14), and their differential activation during auditory verbal imagery might thus be related to defective auditory-verbal recall.

Overall, our data indicate that the propensity to hallucinate in schizophrenia is associated with functional abnormalities in areas implicated in verbal self-monitoring. This is consistent with the notion that auditory hallucinations are derived from defective monitoring of inner $\operatorname{speech}(1,2)$.
Received Oct. 25, 1999; revision received March 16, 2000; accepted April 5, 2000. From the Departments of Biostatistics and Neuroscience and the Division of Psychological Medicine, Section of Neuroimaging, Institute of Psychiatry. Address reprint requests to Dr. Shergill, Division of Psychological Medicine, Institute of Psychiatry, De Crespigny Park, London SE5 8AF U.K.; s.shergill@iop.kcl.ac.uk (email).

Drs. Shergill and Bullmore were supported by the Wellcome Trust.

\section{References}

1. Frith CD, Done DJ: Towards a neuropsychology of schizophrenia. Br J Psychiatry 1989; 153:437-443

2. McGuire PK, Silbersweig DA, Wright I, Murray RM, David AS, Frackowiak RS, Frith CD: Abnormal monitoring of inner speech: a physiological basis for auditory hallucinations. Lancet 1995; 346:596-600

3. Shergill SS, Bullmore E, Simmons A, Brammer MJ, Murray RM, McGuire PK: Auditory verbal imagery: a functional MRI study. Psychol Med (in press)

4. Brammer MJ, Bullmore ET, Simmons A, Williams SCR, Grasby PM, Howard RJ, Woodruff PWR, Rabe-Hesketh S: Generic brain activation mapping in $\mathrm{fMRI}$ : a nonparametric approach. Magn Reson Imaging 1997; 15:763-770

5. Bullmore ET, Suckling J, Overmeyer S, Rabe-Hesketh S, Taylor E, Brammer MJ: Global voxel and cluster tests, by theory and permutation for a difference between two groups of structural MR images of the brain. IEEE Trans Med Imaging 1999; 18:32-42

6. Reisberg D, Wilson M, Smith JD: Auditory imagery and inner speech, in Mental Images in Human Cognition. Edited by Logie R, Denis M. Amsterdam, Elsevier, 1991, pp 59-81

7. McGuire PK, Silbersweig DA, Frith CD: Functional neuroanatomy of verbal self-monitoring. Brain 1996; 119:907-917

8. Shergill SS, Brammer MJ, Williams SR, Murray RM, McGuire PK: Auditory hallucinations: mapping the network involved using functional MRI. Arch Gen Psychiatry (in press)

9. Blakemore SJ, Wolpert DM, Frith CD: Central cancellation of self-produced tickle sensation. Nat Neurosci 1998; 1:635-640

10. Desmond JE, Gabrieli JDE, Wagner AD, Ginier BL, Glover GH: Lobular patterns of cerebellar activations in verbal working memory and finger tapping tasks as revealed by functional MRI. J Neurosci 1997; 17:9675-9685

11. Andreasen NC, Paradiso S, O'Leary DS: “Cognitive dysmetria” as an integrative theory of schizophrenia: a dysfunction in cortical-subcortical-cerebellar circuitry? Schizophr Bull 1998; 24 : 203-218

12. Gray JA, Feldon J, Rawlins JNP, Smith AD: The neuropsychology of schizophrenia. Behav Brain Sci 1991; 14:1-19

13. Silbersweig DA, Stern E, Frith CD, Cahill C, Holmes A, Grootoonk $S$, Seaward J, McKenna P, Chua S, Schnorr L: A functional neuroanatomy of hallucinations in schizophrenia. Nature 1995; 378:176-179

14. Henke K, Weber B, Kneifel S, Wieser HG, Buck A: Human hippocampus associates information in memory. Proc Natl Acad Sci USA 1999; 96:5884-5889 